\title{
Using Virtual Reality to Validate System Models*
}

\author{
Victor L. Winter \\ Department of High Integrity \\ Software Systems Engineering \\ Sandia National Laboratories \\ vlurinte@sandia.gov
}

\author{
Thomas P. Caudell \\ Department of Electrical \\ and Computer Engineering at the \\ University of New Mexico \\ and \\ The Albuquerque High Performance \\ Computing Center \\ tpc@eece.unm.edu
}

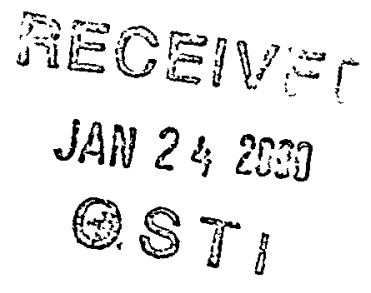

\begin{abstract}
To date most validation techniques are highly biased towards calculations involving symbolic representations of problems. These calculations are either formal (in the case of consistency and completeness checks), or informal in the case of code inspections. We believe that an essential type of "evidence" of the correctness of the formalization process must be provided by (i.e., must originate from) human-based calculation. We further believe that human calculation can be significantly amplified by shifting from symbolic representations to graphical representations. This paper describes our preliminary efforts in realizing such a representational shift.
\end{abstract}

\section{Background}

As science and technology advances, there is an increasing trend to supplement and substitute operator control with automated control. The designs of fullyauomated and semi-automated systems have become more ambitious. The impetus behind these designs to develop systems that are better suited to performing the functions expected/needed of them. For many systems, control possible through software can be more refined, more complex, and can react more quickly than the control that could be provided by an operator. For example, computer controlled fuel delivery systems in automobiles can dramatically increase the fuel efficiency of an engine. Production facilities can

*This work was supported by the United States Department of Energy under Contract DE-AC04-94AL85000. Sandia is a multiprogram laboratory operated by Sandia Corporation, a Lockheed Martin Company, for the United States Department of Energy. be automated using robots, producing more uniform and accurate products, and requiring fewer workers. Automated train controllers can realize complex control algorithms that offer the possibility of increased (1) safety, (2) throughput, (3) energy efficiency, and (1) comfort (e.g., less jerking). Weapons systems can be built that are better suited to achieving their objectives (e.g., minimizing civilian casualties).

All of the systems mentioned above are considered high consequence by their potential customers. It is worth mentioning that high consequence is somewhat of a relative term. Typically, high consequence systems are defined as systems in which there is an extreme negative consquence resulting from failure. For example, a faulty fuel delivery system in an automobile might result in several dearhs leading to a recall of all vehicles having this fuel delivery system. An incorrect robotic manufacturing system can result in destruction of costly equipment (i.e., the robots) as well as an extended shutdown of the manufacturing plant. A faulty train controller could cause the collision of trains, and a faulty weapons system could result in the loss of thousands or even hundreds of thousands of lives.

Typically, a system (high consequence or othernise) is designed, analyzed, implemented, and tested to some extent. At this point. if all has gone well, the physical system itself is tested, if possible. Note that there are many physical systems which, from a reasonable standpoint, cannot be tested. After all, the purpose of testing is to discover failures, and high consequence systems, by definition, should not be allowed to experience failures. For example, it doesn't make sense to "test" a nuclear power plant in hopes of discovering (high consequence) system failures. Also, one-shot systems, like the Arianne 5, cannot be phys- 


\section{DISCLAIMER}

This report was prepared as an account of work sponsored by an agency of the United States Government. Neither the United States Government nor any agency thereof, nor any of their employees, make any warranty, express or implied, or assumes any legal liability or responsibility for the accuracy, completeness, or usefuiness of any information, apparatus, product, or process disclosed, or represents that its use would not infringe privately owned rights. Reference herein to any specific commercial product, process, or service by trade name, trademark, manufacturer, or otherwise does not necessarily constitute or imply its endorsement, recommendation, or favoring by the United States Government or any agency thereof. The views and opinions of authors expressed herein do not necessarily state or reflect those of the United States Government or any agency thereof. 


\section{DISCLAIMER}

Portions of this document may be illegible in electronic image products. Images are produced from the best available original document. 
ically tested.

Because of the difficulties surrounding the physical testing of high consequence systems, the possibility of using computer simulations to test such systems is being explored. The Holy Grail in this field of study is to construct a completely accurate model of reality (i.e., the system and its operating environment) in a virtual setting. If this can be accomplished then virtual testing could be effectively substituted for physical testing thereby eliminating the negative consequences associated with the physical testing of high consequence systems.

\section{The Role of Validation in High Consequence System Development}

Because of the cost associated with their failure, one must provide strong evidence that the design of a high consequence system is fault-free, or failing this, that it is extremely unlikely for the design to contain a fault. The ability of testing alone (physical or simulated) to provide this evidence is highly questionable [1][3][7]. The reason being that testing a suitable portion of the input space would simply take too much time, even if present-day accelerated testing techniques are used. Thus testing will not be sufficiently exhaustive and hence can only serve to validate that system will not experience a failure. In this paper, we use the term validate to describe activities that do not have the potential of promising certainty, and we use the term verify to describe activities that have the potential of promising (mathematical) certainty. For example, exhaustive testing can be used to verify the correctness of a system whereas partial testing can only be used to validate the correctness of a system.

The barrier to providing strong evidence through the partial testing of a system raises an interesting philosophical question: "What is the purpose of testing in the high consequence system arena?" In exploring this question, let us restrict ourselves to high consequence software systems.

When developing high consequence software systems the currently accepted approach consists of two phases. The first phase is called the formalization phase, and the second is called the implementation phase. In the formalization phase, an informal problem description is mapped into a formal framework. The formalization phase is completed when a (formal) specification has been obtained.

In the implementation phase, a formal specifica- tion is used as the basis for producing an (executable) implementation. Since both the specification and the implementation are formal objects (i.e., they have a precise and well-defined semantics) the possibility exists, in theory, to use mathematical proof techniques to formally verify that the implementation satisfies (i.e., is correct with respect to) the specification from which it was derived. Assuming this can be done, what can be said about the implementation? Given these assumptions, the strongest statement that one can make about the implementation is that it does just what specification says it should do. In particular, one cannot say that the implemetation solves the problem it is intended to solve. The reason one cannot make this claim is that the specification irself may be incorrect to begin with.

If verification techniques can be used to demonstrate that an implementation satisfies its specification, then the value added by testing that an implementation satisfies its specification is questionable. In the limit, if we assume flawless and complete verification, then testing, for this phase of software development, becomes redundant.

However, even in this case where one assumes that verification achieves such lofty goals, testing is still necessary because one can never be certain that the formlization phase has been done correctly. This uncertainty arises from the fact that the formalization process is mapping informal knorledge into a formal framework. Thus formalization. by its very nature, lies beyond the reach of verification.

Focusing our attention on the formalization phase. we see that it concerns itself with modeling the real morld using formal notations. Again, in the high consequence realm, we would like to provide strong evidence that these models, upon which the specification is built, are indeed correct. Protiding strong evidence in the correctness of specifications (and the models they are comprised of) is of exireme importance because studies have shown that a significant percentage of implementation errors can be traced back to errors in the specification[6].

To date most validation techniques are highly biased towards calculations inrolving symbolic representations of problems. These calculations are either formal (in the case of consistency and completeness checks), or informal in the case of code inspections. TVe believe that an essential type of "evidence" of the correctness of the formalization process must be prorided by (must originate from) human-based calculation, because the original problem is formulated by humans. We further believe that human calculation can 
be significantly amplified by shifting from symbolic representations to graphical representations. This paper describes our preliminary efforts in realizing such a representational shift.

\section{Models: Mental, Virtual, and Formal}

Within the formalization process, before a model can be specified, it must first be understood by the specifier. This understanding is ultimately in the form of a mental model, and should be as complete as necessary for the context (i.e., with respect to the specification) in which it is to be used. After a mental model has been developed it is then mapped into a formal domain (e.g., mathematical expressions), at which point it becomes part of the system specification.

There are two sources of errors that arise during this activity. First, the specifier can develop an incorrect mental model, and second, the specifier can introduce errors in the process of mapping a (correct) mental model into a formal domain. Both of these types of mistakes can be, to some extent, discovered through consistency and completeness checks. However, it is important to note that consistency and completeness checks are necessary, but not sufficient, to assure that a model is correct.

The two error sources mentioned above are amplified as models become more complex. As the complexity of a model increases the ability for it to be grasped in its totality begins to elude the specifier. When this happens the likelihood of errors occurring can increase dramatically.

We believe that virtual relality can be used to amplify the specifiers ability to construct mental models as well as assist in the mapping of mental models to formal notations. For certain types of system models (e.g., robotic systems) there is a very direct mapping between the model, as described by mathematical formulas, and the physical world. Oftentimes, for these types of systems the mental models can be very clear (though they might be complex) and the major difficulty lies in correctly expressing aspects of these models in terms of symbolic formulas. Research has been conducted on how these kinds of formulas can be written and structured in such a way that they can be understood in an incremental fashion[4]. Our research addresses the same issue, but attempts to solve the problem in a graphical framework rather than in a mathematical framework.

The basic idea is to construct a formally defined rirtual representation of a system. Given such a construction, formulas can be in some sense "projected" onto this system representation. The correctness of a formula can then be validated by human inspection, much in the same way that the mathematically structured symbol-based formulas are validated by human inspection. Our belief is that human intuition and understanding will be much more effective with respect to a $3 D$ visual-based representation of a formula, than it would be in a mathematical domain.

\section{Graphical Representations of Formal Models}

Our long-term research objective is to be able to graphically display (in some suitable manner) aspects of formal (symbolic) descriptions of systems. Currently, our efforts have been restricted to a subset of reactive systems that have been classified as stable [8]. Essentially, the distinguishing features of stable reactive systems are that (1) state transitions can only be initiated by the controller (e.g., not by the environment), (2) transitions between states are deterministic, and most importantly (3) the system can be halted in any state without the need for continuous monitoring and control (i.e., the react process can be suspended in any state).

Stable reactive systems can be formally modeled in the traditional manner as a vector of monitored and controlled variables.

- Let $\vec{m}=\left\{m_{1} \in D_{1}, \ldots m_{j} \in D_{j}\right\}$ denote the observable state of the system.

- Let $\vec{c}=\left\{c_{1} \in C_{1}, \ldots c_{k} \in C_{k}\right\}$ denote the vector of control variables of the system.

- Then $\vec{s}=\left\{m_{1}, \ldots m_{j}, c_{1} \ldots . c_{k}\right\}$ is the state space of the system. Note that $\vec{m} \circ \vec{c}$ contains $D_{1} \times$ $\ldots \times D_{j} \times C_{1} \times \ldots \times C_{k}$ states.

- Transitions between states is generally given by a set of equations of the form:

$$
\begin{aligned}
& -P(\vec{m}) \stackrel{\vec{c}}{\longrightarrow} F(\vec{m}) \text { where } P: \vec{m} \rightarrow \text { bool is a } \\
& \text { predicate on the observable state, and } F: \vec{m} \\
& \rightarrow \vec{m} \text { is a function on the observable state. }
\end{aligned}
$$

We consider a system a suitable candidate for graphical display if there is a sirong (intuitively clear) risual relationship between $D_{1} \times \ldots \times D_{j}$ and physical reality. Robotic systems are often have such properties 
and are therefore prime canditates for the graphical displays we are developing.

Given a model, $\vec{s}=\vec{m} \circ \vec{c}$, of the state space of a system together with a set of transitions we are interested in graphically displaying the following fundamental aspects of the model:

- The observable state space $D_{1} \times \ldots \times D_{j}$ or subsets of the form $\left\{D_{i}, D_{k}, D_{m}\right\}$ where $1 \leq i, k, m \leq j$.

- Subsets of the observable state space defined by predicates (e.g., $P(\vec{m})$ ). We have found it useful to display these subsets in the context of the portion of the observable state space (e.g., $\left.\left\{D_{i}, D_{k}, D_{m}\right\}\right)$ that we are currently viewing.

- Functions $F(\vec{m})$ in terms of an explicit set of input/output pairs. From our experiences we have found that viewing these types of functions in terms of graphical representations of sets, representing the totality of the input output relation defined by the function, is useful.

- Transitions $P(\vec{m}) \stackrel{\vec{c}}{\longrightarrow} F(\vec{m})$. A transition can be displayed by first displaying the subset defined by $P(\vec{m})$ and then using the elements of this set as the input domain for $F$.

In addition to displaying the basic aspects of system models described above, we are also interested in developing a set of operators and a calculus for displaying behavioral aspects of these models. We believe that the following animation operators can be useful for understanding and validating system models:

- Finite repitition of a transition. If $t$ is a transition, then $t^{n}$ denotes $n$ repititions of $t$. Formally, the semantics of finite repitition can be defined as follows:

1. Given a transition of the form: $t=P(\vec{m}) \stackrel{\vec{c}}{\longrightarrow}$ $F(\vec{m})$

2. The expression $t^{n}$ can be displayed as follows:

3. Compute $S_{0}=\left\{\vec{m}_{0} \mid P\left(\vec{m}_{0}\right)\right\}$.

4. while $n \neq 0 \wedge S_{0} \neq \emptyset$ do

(a) Display $S_{0}$.

(b) Compute $S_{1}=\left\{\vec{m}_{1} \mid \exists \vec{m}_{0} \in S_{0} \wedge F\left(\vec{m}_{0}\right.\right.$ ) $\left.=\vec{m}_{1}\right\}$.

(c) Erase the display of $S_{0}$ while smoothly displaying $S_{1}$. (d) $S_{0}:=S_{1}$;

(e) $n:=n-1$;

- Transitive closure of a transition. If $t$ is a transition, then $t^{*}$ denotes the transitive closure of this transition. Formally, the semantics of the transitive closure operator can be defined as follows:

1. Given a transition of the form: $t=P(\vec{m}) \stackrel{\vec{c}}{\longrightarrow}$ $F(\vec{m})$

2. The expression $t^{*}$ can be displayed as follows:

3. Compute $S_{0}=\left\{\vec{m}_{0} \mid P\left(\vec{m}_{0}\right)\right\}$.

4. while $S_{0} \neq \emptyset$ do

(a) Display $S_{0}$.

(b) Compute $S_{1}=\left\{\vec{m}_{1} \mid \exists \vec{m}_{0} \in S_{0} \wedge F\left(\vec{m}_{0}\right.\right.$ ) $\left.=\vec{m}_{1}\right\}$.

(c) Erase the display of $S_{0}$ while smoothly displaying $S_{1}$.

(d) $S_{0}:=S_{1}$;

- Sequential composition of transitions. The sequential composition of transitions $t_{1}, \ldots, t_{n}$ is denoted $t_{1} ; t_{2} ; \ldots ; t_{n}$ and is displayed as follows:

1. $i:=1$;

2. Let $S_{0}$ be the entire observable state space.

3. while $i \leq n \wedge S_{0} \neq \emptyset$ do

(a) Obtain $t_{i}=P_{i}(\vec{m}) \stackrel{\vec{c}}{\longrightarrow} F_{i}(\vec{m})$

(b) Compute $S_{1}=\left\{\vec{m}_{0} \mid \vec{m}_{0} \in S_{0} \wedge P_{i}\left(\vec{m}_{0}\right)\right\}$.

(c) Display $S_{1}$.

(d) Compute $S_{2}=\left\{\vec{m}_{2} \mid \exists \vec{m}_{1} \in S_{1} \wedge F\left(\vec{m}_{1}\right.\right.$ ) $\left.=\vec{m}_{2}\right\}$.

(e) Erase the display of $S_{1}$ while smoothly displaying $S_{2}$.

(f) $S_{0}:=S_{2}$;

(g) $i:=i+1$

- Parallel composition of transitions. The parallel composition of transitions $t_{1}, \ldots, t_{n 2}$ is denoted $t_{1}\left\|t_{2}\right\| \ldots \| t_{n}$ and is displayed as follows:

1. Let $S_{0}$ be the entire observable state space.

2. Compute $S_{1}=\left\{\vec{m}_{0}: \vec{m}_{0} \in S_{0} \wedge \exists i: 1 \leq i \leq\right.$ $\left.n \wedge P_{i}\left(\vec{m}_{0}\right)\right\}$.

3. Display $S_{I}$. 
4. Compute $S_{2}=\left\{\vec{m}_{2} \mid \exists \vec{m}_{1} \in S_{1} \wedge \exists i: 1 \leq i \leq\right.$ $\left.n \wedge F_{i}\left(\vec{m}_{1}\right)=\vec{m}_{2}\right\}$.

5. Erase the display of $S_{1}$ while smoothly displaying $S_{2}$.

- Parallel composition of transition sequences. The parallel display of transition sequences becomes problematic without considering timing aspects. We are presently researching ways to display these types of expressions.

\subsection{Display Issues}

Our present approach is to display the observable state space as a collection of transparent geometric objects (mostly cubes) that stand in some geometric relationship to one another (e.g., touching, overlapping, etc.). In this approach, a geometric object represents represents a portion of the observable state. More precisely, geometric objects are defined by coordinate vectors that map directly onto subvectors of $\vec{m}$.

For example, consider a robotic system consisting several components including an electro-magnet which can be moved to various coordinates in the $x-y$ plane. Suppose that there exists two sensors, $m_{1}$ and $m_{2}$, that respectively monitor the $x$-coordinate and the $y$ coordinate of the electro-magnet. If the magnet can be moved to three distinguishable $x$-coordinates and two distinguishable $y$-coordinates, then the observable state space of the system can be modeled as follows:

- $\vec{m}=\left\{m_{1} \in D_{1}, m_{2} \in D_{2}, \ldots m_{j} \in D_{j}\right\}$

- where

$-D_{1}=\{1,2,3\}$

- $D_{2}=\{1,2\}$

- $D_{3} \ldots D_{j}$ are the domains of the monitored variables for the rest of the system (which we are not presently interested in).

A crude $2 D$ representation of the state space of the electro-magnet is simply:

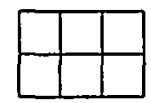

If we assume the traditional orientation of the $x$ and $y$ axes, then geometric object (i.e., the square) in the upper right corner denotes the subvector of $\vec{m}$ consisting of the monitored variables $m_{1}$ and $m_{2}$ having the values 3 and 2 respectively.
In general, the Euclidean relationships that objects need to satisfy are determined by the corresponding relationships present in the physical system. It is in this manner that objects in the model are bound to (and reflect attributes of) the physical system.

As models get more complex it becomes useful to visually focus on portions of the model (e.g., subsets of objects). One way to support this is to provide a mechanism for making objects invisible, transparent, or wire framed.

\subsubsection{Displaying Predicates}

It should be noted that there are various issues that need to be addressed in order to define what it means to display the states "satisfied" by a predicate. Consider a system whose observable state space is modeled by two monitored variables. That is, $\vec{m}=$ $\left\{m_{1} \in D_{1}, m_{2} \in D_{2}\right\}$. Further more, suppose that $D_{1}=\{1,2,3\}$ and $D_{2}=\{1,2\}$, and that $D_{1} \times D_{2}$ is displayed as a $3 \times 2$ array of cubes, whose $2 \mathrm{D}$ version is shown below:

\begin{tabular}{|l|l|l|}
\hline clear & clear & clear \\
\hline clear & clear & clear \\
\hline
\end{tabular}

Consider displaying the predicate $P(\vec{m})=m_{1}<3$ by coloring those objects that satisfy the predicate, and rendering invisible the objects that do not satisfy the predicate.

\begin{tabular}{|l|l|l|}
\hline opaque & opaque & clear \\
\hline opaque & opaque & clear \\
\hline
\end{tabular}

The relationship betwen the states defined by the predicate and display seems intuitive. However, now let us consider a different geometrical relationship. Suppose that $m_{1}$ denotes the state of a conveyor belt having three regions, left, middle, and right, and that $m_{2}$ denotes the state of an adjacent conveyor belt having two regions, left and right. If the right region of the first conveyor is adjacent to the left region of the second conveyor, we get the following $2 \mathrm{D}$ representation:

\begin{tabular}{|l|l|l|}
\hline clear & clear & clear \\
\hline
\end{tabular}

Again, let us consider the predicate $P(\bar{m})=m_{1}<3$. At this point, there are two possible displays.

\begin{tabular}{|c|c|c|c|c|}
\hline opaque & opaque & clear & opaque & opaque \\
\hline
\end{tabular}

or 


\begin{tabular}{|c|c|c|c|c|}
\hline opaque & opaque & clear & clear & To \\
\hline
\end{tabular}

Given the discription of the physical, two conveyor, system, we believe that the second display more clearly shows the area that the predicate is intended to describe. The reason being that the state space of the second conveyor is in no way restricted by the predicate. In fact, from the perspective of the predicate, the second conveyor could be removed altogether (i.e., there is correlation between the objects denoting the second conveyor and those denoting the first). From these observations, our algorithm for displaying predicates is as follows:

1. Given a vector of monitored variables, $\vec{m}=\left\{m_{1} \in\right.$ $\left.D_{1}, \ldots m_{j} \in D_{j}\right\}$, group these variables according to the physical components which they correspond to. For example, all variables used to describe the state of a conveyor belt are grouped together.

2. Given a predicate $P$ determine the set of monitored variables (e.g., $\left.\left\{m_{1}, m_{4}, m_{5}\right\}\right)$ that are referenced in the predicate. For example, the predicate in the example above references the set of variables $\left\{m_{1}\right\}$.

3. Given a referenced set of monitored variables, determine which components these variables belong to. These are the components that are referenced by the predicate.

4. Display all the variables belonging to all of the components that are referenced by the predicate.

Given the above display algorithms, we must now turn to the graphical environment in whcih the state spaces will be rendered. The following section briefly describes our virtual environment system.

\section{The Flatland Virtual Envi- ronment Shell}

Flatland, named in honor of Edwin Abbott's 1884 book by the same name, was written in the first half of 1999 to serve as a platform for our virtual reality research. Flatland [2] is a portable, cross platform Irix/Unix/Linux based multithreaded $\mathrm{C} / \mathrm{C}++$ based system that uses the graphics language OpenGL (or MESA) and associate libraries, including the GLUT library. A virtual environment is built from objects linked together in a world or scene graph, much like VRML or Open Inventor. Flatland has been designed to support a wide sensorium, including 3D graphics, 3D sound, 3D smell, simple haptics, and verbal conversations, both with artificial elements in the environment and other immersed workers. Each user operates from within their personal-workspace represented as a surrounding dodecahedral vessel, which is flown through the VE and contains customizable consoles, screens, and controls, as well as their personal tool chest. The worker interacts with the vessel and environment through the use of custom hand held wands and the use of voice commands.

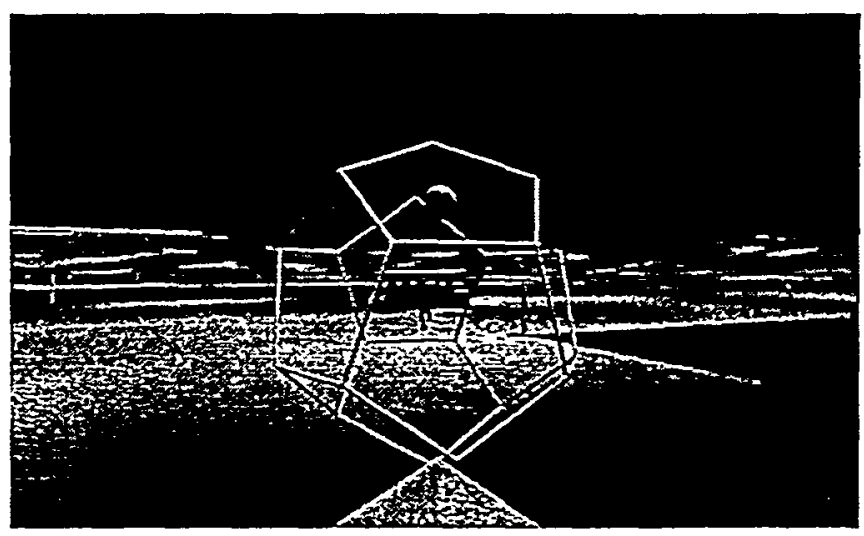

A View of Flatland

\section{Example: A Robotic System}

To date, we have used a robotic system, called the production cell, as a vehicle for trying out and developing our ideas regarding the use of visual models. The production cell consists of 2 conveyor belts, an elevating rotating table, a robot having tro arms, a press, and a crane. A detailed description of the production cell can be found in [5].

The objective of the production cell is to process metal plates. A plate is processed in the following manner: First, it is placed on the first conveyor belt (called the feed belt), which then transfers it to the elevating rotating table. The table then changes its postion so that arm 1 of the robot can pick up the plate using an electro-magnet. From there the plate is moved into the press where it is "processed". Then, arm 2 of the robot removes the plate from the press and places it on the second conveyor belt (called the deposit belt) which transfers it to the crane. The crane takes the plate and places it on the feed belt and the cycle continues. 


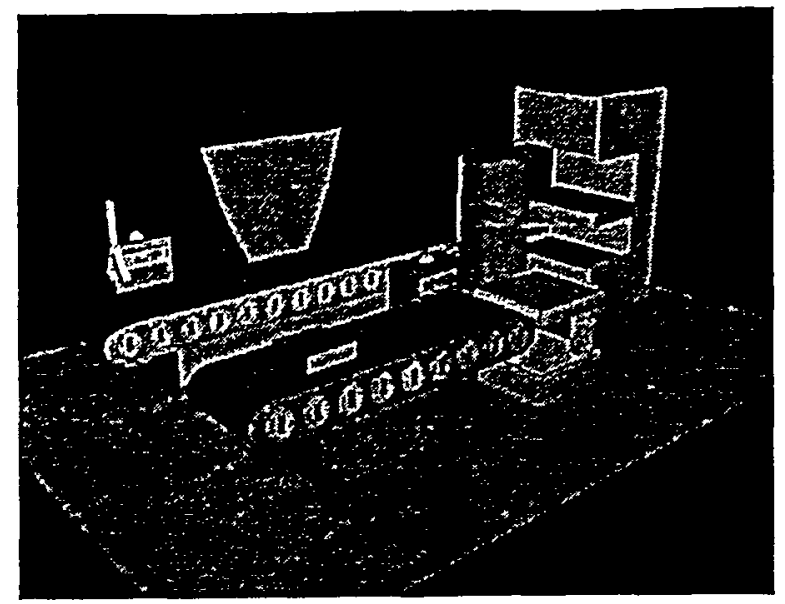

The Production Cell

In addition to the behavior described in the previous paragraph the production cell is also capable of numerous failures, which are considered to be of high consequence. These failures result in damaged system components which are expensive to replace and which prevent the plant from processing plates.

The observable (stable) state of the production cell can be formally described by a vector of 25 monitored variables, $\vec{m}=\left\langle m_{1}, m_{2}, \ldots m_{25}\right\rangle$. Some of the system attributes described by these variables are:

- The rotation and height of the elevating rotating table.

- The presence/absence of a plate on the rotating table.

- The presence of a plate on the left and right regions of a conveyor belt.

- The rotational position of the robot and the extensions of both of its arms.

- The state of the electro-magnet on the robot arms, as well as whether the arm is holding a plate.

Each of these variables is quantified over a specific domain. For example, a variable denoting the presence/absence of a plate on the rotating table is quantified over a domain having two values (e.g., $\{$ true,false $\})$. The variable denoting the angle of rotation of the elevating rotating table is quantified over the set of possible angles that this sensor can distinguish.

Modeling sensor data in its concrete form can result in variables being quantified over very large sets (e.g., containing hundreds or even thousands of elements). However, most of these values are not interesting from the perspective of the specification, and for this reason it is possible to formally group sets of elements into equivalence classes. Intuitively, two elements belong to the same equivalence class, if and only if the same control vector can be applied, in all cases, to both elements in order to achieve the desired overall system behavior.

Grouping elements into equivalence classes results in the corresponding monitored variable being quantified over an abstracted domain. For example, the concrete sensor data describing the angle of rotation of the elevating rotating table can be abstracted to a domain having six values. This abstracted domain defines the equivalence classes that need to be distinguished by a controller of this system in order to achieve the behavior described in the specification.

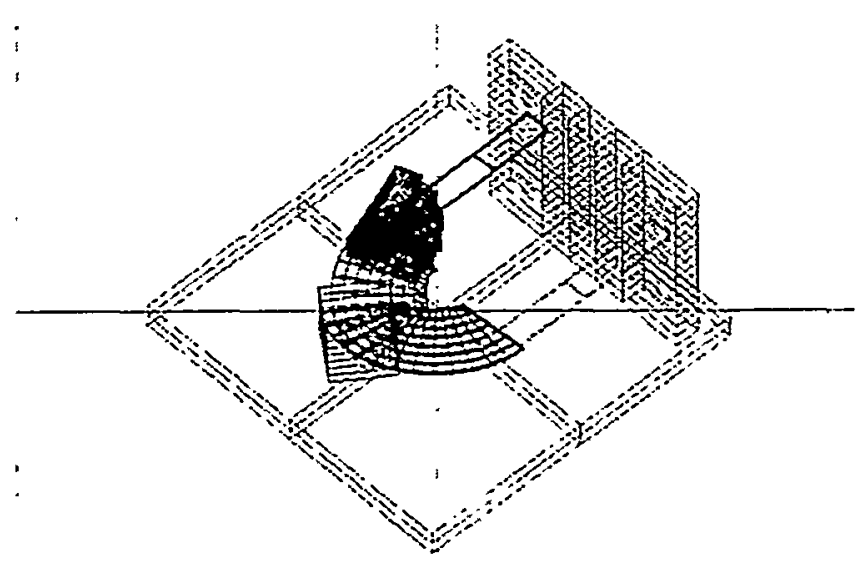

A view of the spacial relationships in the abstracted state space of the Production Cell

Given a model of the observable state space of this system, we next define hor the assignment of values to controlled variables can change the system state. In its most fundamental form one would explicitly define (i.e., enumerate) all possible transitions from every single observable state. However, such an approach would lead to a system where the number of transitions is proportional to the size of the observable state space. Even for modest sistems, the observable state space can contain on the order of $10^{10}$ states, making an exhaustive enumeration approach infeasible. For this reason, state transitions are written in more compact and general terms. This has the unfortunate consequence of increasing their complexity as well as the likelihood that ther contain one or more errors. Abstractly, transitions are of the form: 


$$
P(\vec{m}) \stackrel{c_{i}=v_{j}}{\longrightarrow} f(\vec{m})
$$

where $P$ is a predicate on the observable state, $c_{i}=v_{j}$ denotes the assignment of the value $v_{j}$ to the control variable $c_{i}$, and $f: \vec{m} \rightarrow \vec{m}$. In practice, the notation we use is slightly different and we include the expression above to highlight the three aspects of a transition: (1) a boolean expression on the vector of monitored variables, (2) the assignment of a value to a control variable, and (3) a function defining a change of the monitored variables.

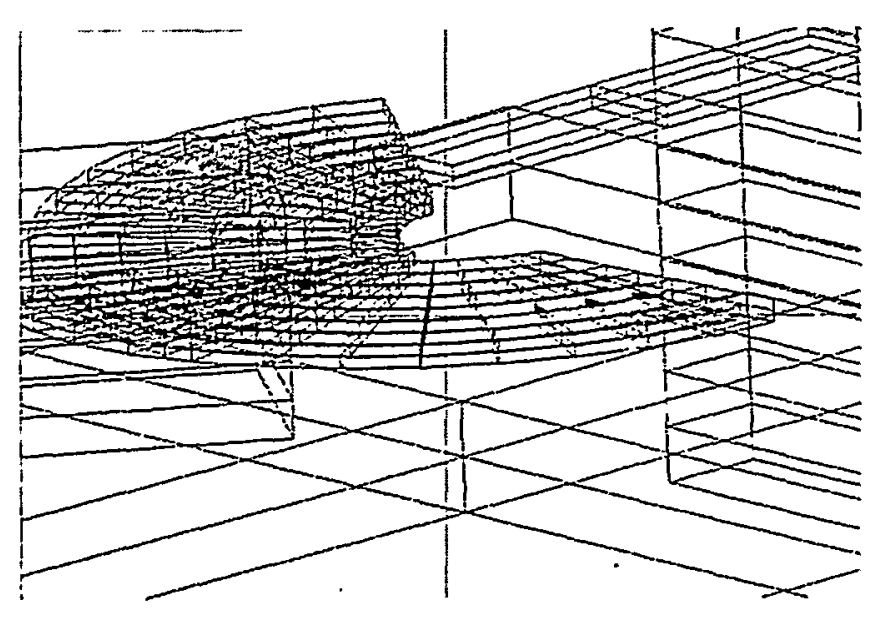

A more detailed view of of the spacial relationships in a portion of the abstracted state space of the Production Cell

It is at this point, when we are defining transitions, that mapping our understanding (i.e., our mental model) of the system into symbolic expressions (i.e., transitions) can become difficult. This difficulty arises due to the complexity that is introduced by the interaction between system components. For example, if $\operatorname{arm} 1$ of the robot has a particular range of extension, then rotating the robot can result in a collision with the press, if the press is in a particular position. From this example, we see that from the perspective of safety, component interaction restricts the state space of components and creates dependencies between the domains that monitored variables are quantified over.

That is, the values that a monitored variable can have is constrained by properties of other monitored variables (e.g., the monitored variable $m_{i}$ can only have value $r$ when the monitored variable $m_{j}$ has a value that is less than $s$ ).
We now consider a slightly simplified example of an actual transition from the production cell. We consider the definition of a particular set of conditions under which the (elevating rotating) table can be elevated. The complexity in real problem from which this example is derived arises from the spacial dependencies that exist between arm 1 of the robot and the table. For example, if arm 1 is in a certain position and is holding a plate and there is a plate on the table, then under certain circumstances a plate collision will occur when the table is elevated to its uppermost position. Another issue that needs to be considered is the effect of having the electro-magnet of arm 1 "on" when it is in close proximity to the table. If arm 1 is too close to the table, then having the electro-magnet "on" will cause problems when the table has a plate on it.

In this example, we are intested in defining the state space of arm 1 in which collisions with the table are not possible. That is, the state space of arm I that does not intersect with the state space of the table. We will also assume (for simplicity) that in this region, the state of the electro-magnet cannot attract a plate that is on the table.

We begin by formally defining the abstracted observable state space of the table and the robot.

Table $=\left\langle x_{1}, x_{2}, x_{3}\right\rangle$ and

Robot $=\left\langle x_{4}, x_{5}, x_{6}, x_{7}, x_{8}, x_{9} . x_{10}\right\rangle$ and

$\forall i: 1 \leq i \leq 10 \rightarrow x_{i} \in D_{i}$ where

$D_{1}=\{1,2,3\}=$ height of table

$D_{2}=\{1,2, \ldots, 6\}=$ angle of rotation of table

$D_{3}=\{0,1\}=$ presence/absence of plate on table

$D_{4}=\{1,2, \ldots, 19\}=$ angle of rotation of robot

$D_{5}=\{1,2, \ldots, 8\}=$ extension of arm 1

$D_{6}=\{0,1\}=$ arml magnet off/on

$D_{i}=\{0,1\}=$ presence/absence of plate for arm 1

$D_{3}=\{1,2, \ldots, 7\}=$ extension of arm 2

$D_{9}=\{0,1\}=$ arm2 magnet off/on

$D_{10}=\{0,1\}=$ presence/absence of plate for arm 2

We now combine the formal description of the abstracted state space of our system with spacial knowledge. In general, we are interested in the spacial relationships that states have to one another. In this example, we are specifically interested in the intersection of the state spaces describing the table and arm 1 of the robot.

Given the appropriate spacial information we next construct a graphical representation corresponding to the formal description given above. After this has 
been accomplished we can consider projecting a predicate onto the graphical representation of the model in the manner described in Section 4.1.1.

We are interested in displaying the following predicate:

$$
\begin{gathered}
P\left(x_{1}, x_{2}, x_{3}, x_{4}, x_{5}, x_{6}, x_{7}, x_{8}, x_{9}, x_{10}\right)= \\
\left(x_{4} \leq 8 \vee x_{5} \doteq 1\right) \\
\wedge \\
x_{1}<3 \wedge x_{3}=1
\end{gathered}
$$

This predicate describes (1) arm 1 of the robot which is in a rotational or extensional position that does not intersect with the uppermost position of the table, and (2) a table containing a plate which is not in its uppermost position. The figures below show two views of the model with this predicate displayed. Note that the first view emphasizes $\left(x_{4} \leq 8 \vee x_{5}=1\right)$ while the second emphasizes $x_{1}<3 \wedge x_{3}=1$. Because orientation plays such a pivital role in the cognition of complex models it is very useful to be able to "fly around" a model.

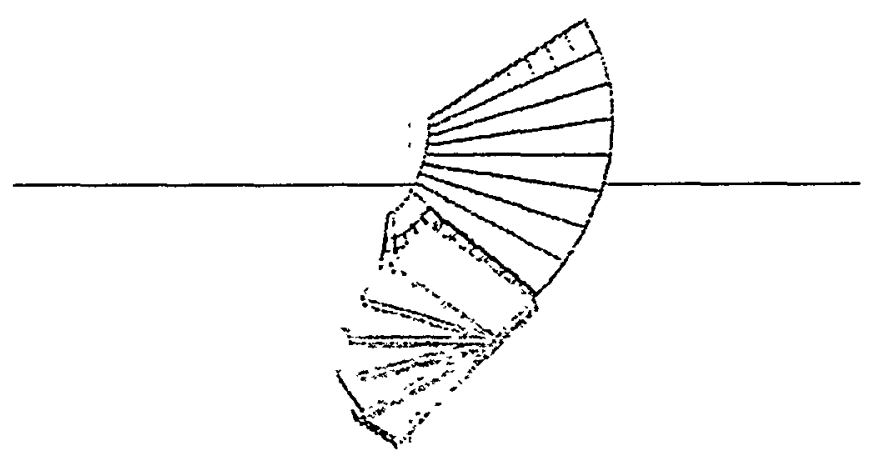

A view emphasizing $\left(x_{4} \leq 8 \vee x_{5}=1\right)$

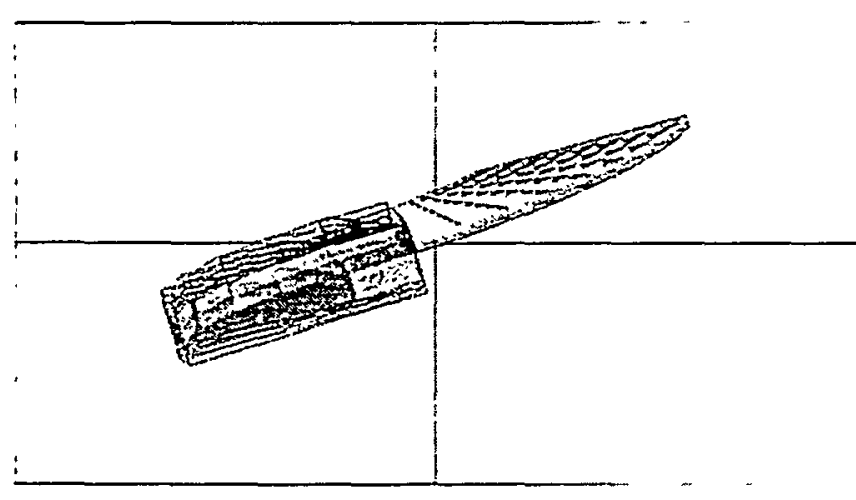

A view emphasizing $x_{1}<3 \wedge x_{3}=1$
Viewing this predicate helps us to validate that it describes the intended portion of the state space. After validation, this predicate then can be used as a precondition defining a state transition, such as the one given below.

$$
\begin{aligned}
& \text { system( } \\
& \text { table }\left(x_{1}, x_{2}, x_{3}\right) \text {, } \\
& \operatorname{robot}\left(x_{4}, x_{5}, x_{6}, x_{\overline{7}}, x_{8}, x_{9}, x_{10}\right) \text {, } \\
& \text { ) } \\
& : x_{1}<3 \wedge x_{3}=1 \\
& \wedge \\
& \left(x_{4} \leq 8 \vee x_{5}=1\right) \\
& \rightarrow \text { table_up } \rightarrow \\
& \operatorname{system}\left(\operatorname{table}\left(x_{1}+1, x_{2}, x_{3}\right)\right. \text {; } \\
& \operatorname{robot}\left(x_{4}, x_{5}, x_{6}, x_{7} . x_{8}, x_{9}, x_{10}\right) \text {, }
\end{aligned}
$$

In the above transition, the "system" consists of a table and a robot. Monitored variables describing aspects of the table are grouped with the table, and monitored variables describing aspects of the robot are grouped with the robot. Recall that this grouping is necessary in order to properly display predicates ${ }^{1}$ (see Section 4.1.1). If a system state satisfies the predicate $x_{1}<3 \wedge x_{3}=1 \wedge\left(x_{4} \leq 8 \vee x_{5}=1\right)$, then the table $u p$ command (i.e., setting the proper control variable to this value) will cause the system to transition to a state where the value of $x_{1}$ is incremented by 1 and all other monitored variables remain unchanged.

\section{Conclusions and Future Work}

In the high consequence realm. demonstrating the correctness of the formalization phase has not been satisfactorily addressed by current validation techniques. Furthermore, we believe that human-based validation techniques based on reasoning about symbolic representations of systems do not effectively take advantage of the human cognitive process. We also believe that suitable visual and spacial representations of information can be more readily understood by humans.

A significant number of systems have physical characteristics that make them suitable candidates for graphical validation techniques based on the ideas described in this paper. At present, we have developed

\footnotetext{
${ }^{1}$ Actually, a more refined grouping is needed to generate the display shown in this example.
} 
an initial theory for displaying such systems in a virtual environment. We also have developed a prototype system to try out and refine our ideas.

A deficiency in the current system is that models and their spacial relationships are created by hand. We have developed a prototype version of a specification language for formally describing spacial relationships and constraints that a virtual model must satisfy. These spacial relationships and constraints defined in a specification can generally be satisfied by multiple graphical interpretations. Allowing multiple graphical interpretations is useful because it permits a graphical interpretation to be altered (while still preserving specified properties) to enhance understandibility. However, it complicates the task of automatically generating graphical interpretations. It is one of our long term research goals to address this problem in a suitable manner.

In the short term, we would like to integrate our system with models that have been rigorously constructed, such as Pro-E models. We belive that the integration of such models into our system would be a significant step in ensuring that our displays faithfully. represent aspects of the physical system.

\section{Acknowledgements}

We would like to give special thanks to Shelly Richards for her efforts in developing the prototype of the system described in this paper.

\section{References}

(1) Ricky W. Butler and George B. Finelli. The Infeasibility of Quantifying the Reliability of LifeCritical Real-Time Software.

[2] Thomas P. Caudell. For more on Flatland, see: uruw.unm.ahpcc/homunculus.

[3] C. Michael Holloway. Why Engineers Should Consider Formal Methods.

[4] R. Janicki, D. Parnas, and J. Zucker. Tabular Representations in Relational Documents. CRL Report No. 313, November 1995.

[5] C. Lewerentz and T. Lindner. Formal Development of Reactive Systems: Case Study Production Ceill. Lecture Notes in Computer Science Vol. 891, Springer-Verlag.
[6] R. R. Lutz. Analyzing Software Requirements Errors in Safety-Critical, Embedded Systems.

[7] John Rushby. Formal Methods and their Role in the Certification of Critical Systems.

[8] Victor Winter. A Synchronous Paradigm for Modeling Stable Reactive Systems. Proceedings of the $4^{\text {th }}$ International Symposium on High Assurance Systems Engineering (HASE), November 1999. 